\title{
Thoracoscopic management of incarcerated lung herniation after blunt chest trauma: a case report and literature review
}

\author{
Ting-Yen Chiang ${ }^{1}$, Ming-Fang Yin $^{1}$, Shun-Mao Yang ${ }^{1,2}$, Ke-Cheng Chen ${ }^{1}$ \\ ${ }^{1}$ Division of Thoracic Surgery, Department of Surgery, ${ }^{2}$ Department of Surgery, National Taiwan University Hospital, Hsin-Chu Branch, Hsinchu \\ City, Taiwan \\ Correspondence to: Shun-Mao Yang, MD. Department of Surgery, National Taiwan University Hospital, 7 Chung-Shan South Road, Taipei, Taiwan. \\ Email: mutayang@gmail.com.
}

\begin{abstract}
Traumatic lung herniation is a rare condition that is usually treated with thoracotomy and pericostal fixation of adjacent ribs with sutures. We described a traumatic lung herniation case successfully managed with hernia reduction and double-layered repair of the intrathoracic defect, completed via a total thoracoscopic approach.
\end{abstract}

Keywords: Chest trauma; lung herniation; thoracoscopic surgery

Submitted Nov 11, 2016. Accepted for publication Feb 08, 2017.

doi: $10.21037 /$ jtd.2017.03.41

View this article at: http://dx.doi.org/10.21037/jtd.2017.03.41

\section{Introduction}

Lung herniation is a protrusion of the lung parenchyma beyond the normal boundary of the thoracic cavity (1). It is a rare condition, and most cases result from trauma (penetrating or blunt) or inadequate closure of the chest wall after thoracic surgery. Treatment of a lung hernia depends on symptoms, location, and size; there is no standard management of lung herniation. Since asymptomatic hernias generally remain asymptomatic, and do not change, they may not require treatment (2). For symptomatic lung herniation, surgical repair is generally recommended, if no surgical contraindications exist. Thoracotomy is more widely used than the thoracoscopic approach. Pericostal fixation of adjacent ribs with sutures is the most common way to repair chest wall defects; however, several other methods had been reported (3). We describe a case of traumatic lung herniation surgically repaired via the thoracoscopic approach. We repaired the chest wall defect with hernia mesh and an inner lining surgical membrane. We discuss and compare recent surgical treatments of traumatic lung herniation.

\section{Case presentation}

A 64-year-old man, with no history of systemic disease, was hit by a motor vehicle, and presented to our emergency room complaining of left knee and right hand pain. Physical examination revealed local tenderness and swelling of the left leg, limited range of motion due to pain, and an abrasion. Lower extremity computed tomography (CT) showed a non-displaced fracture of the medial condyle of the tibia, and a fibular neck fracture. Chest radiography (Figure 1) showed prominent bilateral lung markings. No other respiratory symptoms were complained and his vital sign was stable, no desaturation or tachypnea was presented, thus, no further examination was performed. The patient was discharged after application of a long leg cast. The patient presented to our ER three days later complaining of hemoptysis, dyspnea, chest pain, and swelling of the left chest. A $10 \mathrm{~cm} \times 10 \mathrm{~cm}$ soft, mildly tender mass was identified over left chest wall. Erythema and ecchymosis were not identified in this area. Chest radiography showed ground glass pattern in the left lung, particularly in the left upper lobe, and subcutaneous emphysema in the neck and left axillary area (Figure 2). We reviewed his first Chest radiography we took three days ago and a subtle fracture over left 2 nd fib was found. Chest CT showed the lung protruding through a chest wall defect, and a hemothorax (Figure 3). The patient underwent emergency surgery for lung herniation. Under general anesthesia, with 


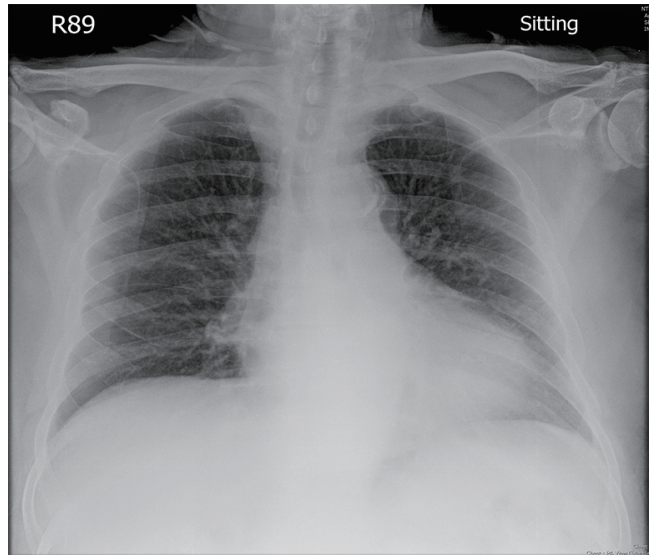

Figure 1 Chest radiography performed at the first admission. Prominent bilateral lung markings and subtle fracture of left second rib were noted.

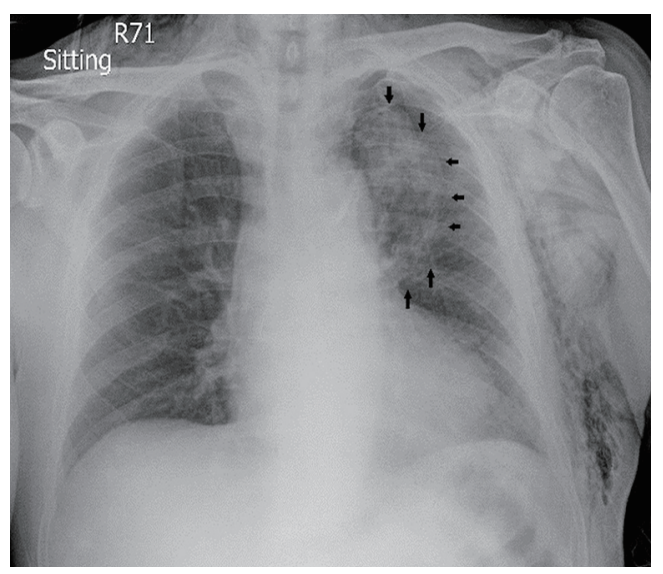

Figure 2 Chest radiograph performed three days after trauma shows ground glass pattern in the left upper lung field (arrows) and subcutaneous emphysema in the neck and left axillary area.

double-lumen intubation and single lung ventilation, the patient was positioned in right decubitus position. The thoracoscopy was placed into the eighth intercostal space at the midaxillary line; a working port was placed in the seventh intercostal space in the auscultatory triangle; and an anterior working port was placed in the fifth intercostal space. Under thoracoscopy, partial herniation of the left upper lobe through the anterior chest wall defect was identified (Figure 4A). Disruption of the visceral pleura, with focal congestion, was noted on the herniated lung parenchyma (Figure 4B). The herniated lung was reduced, and a wedge resection of the diseased lung parenchyma was

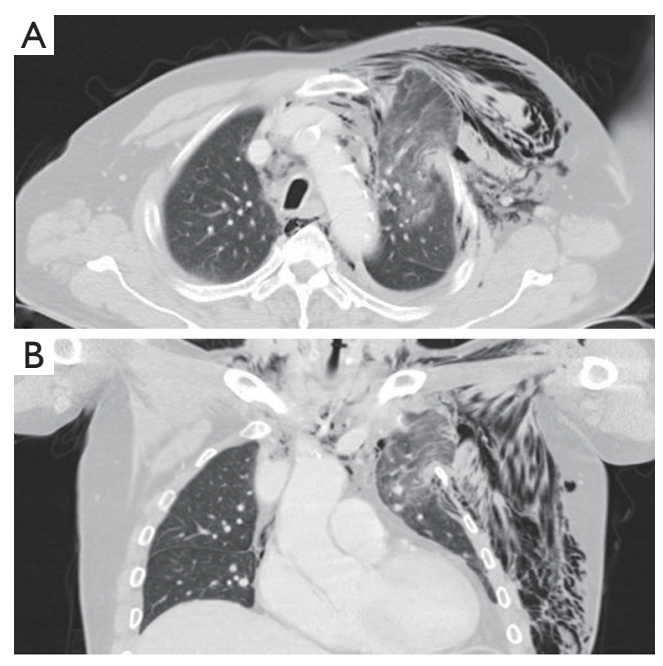

Figure 3 Computed tomography performed three days after trauma shows herniation of the left upper lung with pneumomediastinum and a small left pneumothorax. Soft tissue emphysema was also evident at the left chest wall. A, axial view; B, coronal view.

done. The $4 \mathrm{~cm} \times 5 \mathrm{~cm}$ chest wall defect (Figure 4C) was repaired with a hernia mesh (BARD ${ }^{\mathrm{TM}}$ Mesh, Davol Inc.), and an inner side attached surgical membrane (Gore-Tex ${ }^{\circledR}$ Surgical Membrane, W.L. Gore \& Associates, Inc.) was fixed with titanium spiral tacks (Auto Suture ProTack ${ }^{\mathrm{TM}}$ Fixation $5 \mathrm{~mm}$, Covidien; Figure 4D). A 28-French chest tube was placed via the camera port. After surgery, the patient was admitted to the intensive care unit. The patient was extubated on the second postoperative day, and the chest tube was removed on the eighth postoperative day. The hospital course was uneventful, and the patient was discharged in stable clinical condition, two weeks after the operation. A chest radiograph, obtained in the outpatient department, one month after surgery showed clear lung fields and resolution of subcutaneous emphysema (Figure 5). There was no recurrence during the one year of follow-up.

\section{Discussion}

Lung herniation was first reported in 1499 by Roland (4). While lung herniation can be congenital or acquired, $80 \%$ of lung hernias are acquired (5). Acquired lung herniation is generally related to trauma, but can be spontaneous, or caused by local pathologic conditions (6). Lung herniation is suspected clinically, and confirmed via chest radiography or CT (2).

There is no consensus on the managements of lung 

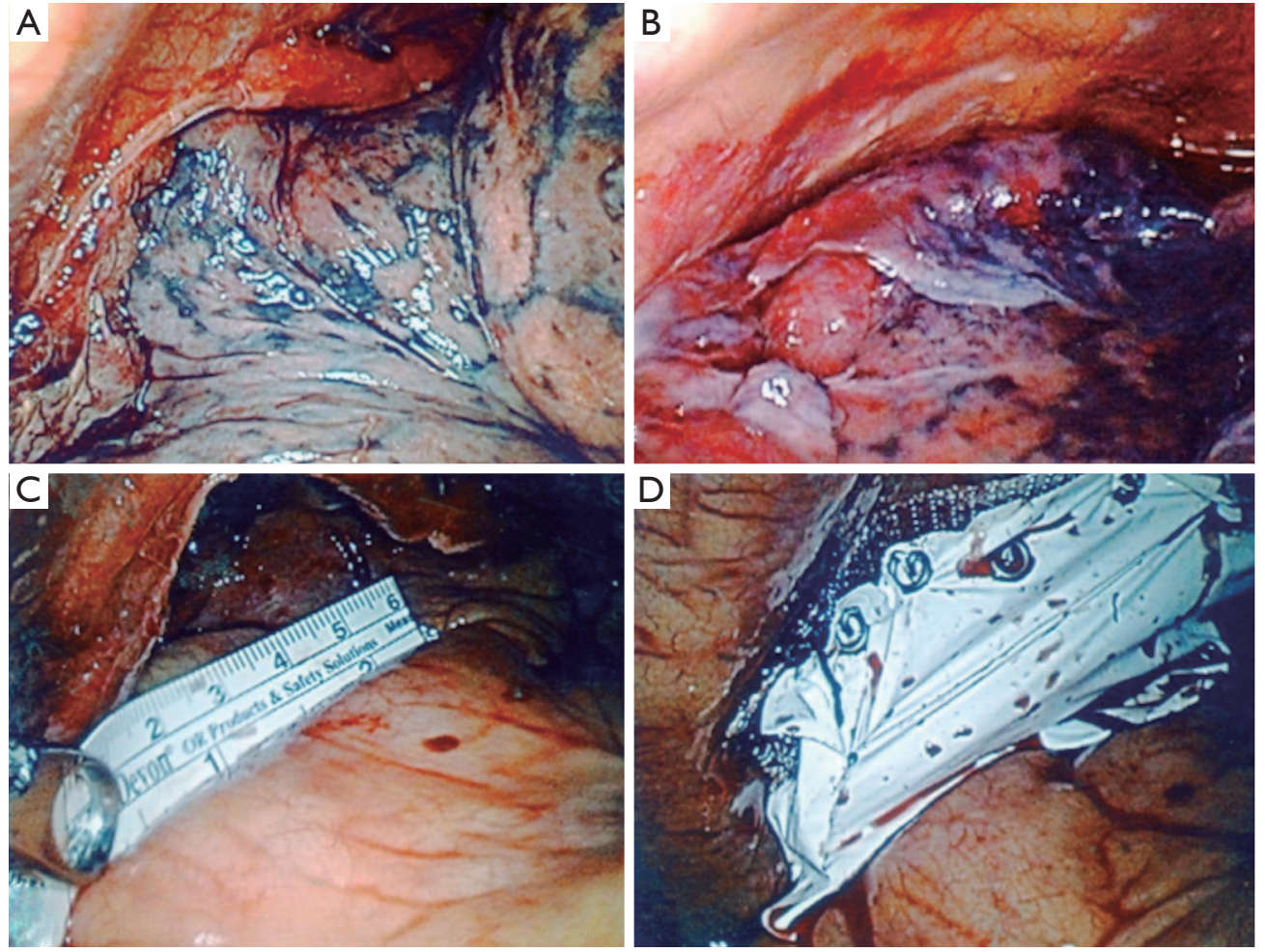

Figure 4 The thoracoscopic findings. (A) Left upper lobe partial herniation into anterior chest wall defect; (B) disruption of visceral pleura with focal congestion was noted after reduction of the herniated lung parenchyma; (C) the chest wall defect, $4 \mathrm{~cm} \times 5 \mathrm{~cm}$ in size; (D) the defect was repaired using a hernia mesh with inner attached surgical membrane, fixed with titanium spiral tacks.

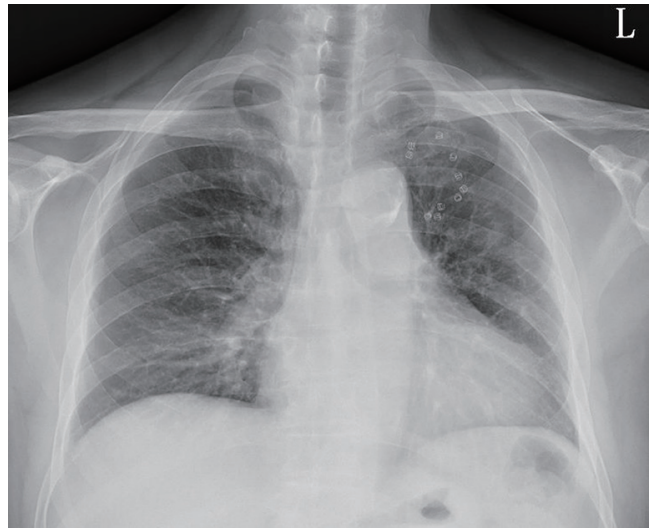

Figure 5 The chest radiograph obtained one month after surgery.

herniation. The treatment can be conservative, or a surgical repair. Conservative treatment includes compression with pads and corsets, treating the underlying cause, and weight loss (7). However, chest wall compression is associated with limited thoracic wall motion, reduced pulmonary compliance, and the risk of atelectasis and infection (3). Wani et al. presented a case of spontaneous intercostal lung herniation complicated by rib fractures. Because of the patient's high surgical risk, the case was managed conservatively. However, this patient experienced poorly controlled chest pain and shortness of breath, and the herniation progressed both clinically and radiologically, suggesting that surgical intervention is ideal if clinically feasible (1). Surgical decisions are made based upon the size of the hernia, the symptoms (pain, dyspnea), the risk of parenchyma incarceration (for example, difficult hernia reduction or small chest wall defect), and the patient's comorbid conditions $(7,8)$. In reported cases, the surgical techniques varied, and included direct closure of the defect, and closure with mesh or muscle flaps. Fackeldey et al. (3) reported that the most commonly used surgical method, after resecting the incarcerated lung parenchyma, is pericostal fixation of the adjacent ribs with absorbable 
sutures, via thoracotomy. For smaller defects, pericostal fixation is useful, but larger defects require reconstruction of the chest wall, using surrounding tissues or synthetic materials (7). Plastic surgical procedures, such as fascia lata grafts or muscle flaps, are seldom used in large thoracic wall defects. There are nonabsorbable (polypropylene; Prolene, Marlex, and expanded polytetrafluoroethylene; ePTFE, Gore-Tex) and absorbable (polyglactin; Vircyl) meshes that can be used in the repair of lung herniation. However, complications, such as fistula formation, seroma, and infection, had been reported with the use of meshes (3).

Most lung hernia repairs are performed via thoracotomy; however, there are several case reports demonstrating successful management using thoracoscopic assists (8-13). Owing to the difficulty of repairing the bony defect of chest wall from inside of thorax, most thoracoscopically managed cases are of a hybrid nature, and the important procedure of repair/reconstruction is done under direct visualization, through a mini-thoracotomy with thoracoscopy guidance. Spiral tacks, which are commonly applied in laparoscopy inguinal hernia repair, have been used for the management of lung hernia $(9,13)$, and improve the ease of intracorporeal fixation. Van Den Bossche et al. (9) first described this method in a case of spontaneous intercostal lung hernia, which was repaired by using a polypropylene mesh. Hebra et al. (13) reported a pediatric patient, with a delayed onset handlebar intercostal hernia, 10 days after blunt chest trauma, managed with elective thoracoscopy. The defect was covered by using a Gore-Tex patch, which was fixed with multiple spiral tacks. No recurrence noted after one year of follow-up. In our case, there was no remaining intercostal muscle or fascia. Therefore, we used a duallayered material to provide strength to the repair, and to prevent pleural adhesion.

\section{Conclusions}

In this case, the patient presented after a traumatic injury, complaining of pain and hemoptysis. Clinical and radiographic findings indicated the patient had a lung hernia. We successfully used a total thoracoscopic approach, with hernia mesh and surgical membrane, to repair the traumatic lung herniation. At the one-year follow-up, the patient reported no associated symptoms, and there was no evidence of recurrent herniation. We used a double-layer repair, which is stronger than either direct repair or the use of a surgical membrane. We suggest that, under the right circumstances, thoracoscopic surgery is a safe and effective method of treating traumatic lung herniation.

\section{Acknowledgements}

None.

\section{Footnote}

Conflicts of Interest: The authors have no conflicts of interest to declare.

Informed Consent: Written informed consent was obtained from the patient for publication of this case report and any accompanying images.

\section{References}

1. Wani AS, Kalamkar P, Alhassan S, et al. Spontaneous intercostal lung herniation complicated by rib fractures: a therapeutic dilemma. Oxf Med Case Reports 2015;2015:378-81.

2. Weissberg D. Lung hernia - a review. Adv Clin Exp Med 2013;22:611-3.

3. Fackeldey V, Junge K, Hinck D, et al. Repair of intercostal pulmonary herniation. Hernia 2003;7:215-7.

4. Roland. De pulmonis sanarpot liber III (cap) XXV. In: De Chavliae G. Cyrugia 1499:144.

5. Moncada R, Vade A, Gimenez C, et al. Congenital and acquired lung hernias. J Thorac Imaging 1996;11:75-82.

6. Forty J, Wells FC. Traumatic intercostal pulmonary hernia. Ann Thorac Surg 1990;49:670-1.

7. Kara HV, Javidfar J, D'Amico TA. Spontaneous herniation of the lung and diaphragm treated with surgical repair. Ann Thorac Surg 2015;99:1821-3.

8. Reardon MJ, Fabré J, Reardon PR, et al. Video-assisted repair of a traumatic intercostal pulmonary hernia. Ann Thorac Surg 1998;65:1155-7.

9. Van Den Bossche MR, Leman G, Ballaux KE, et al. Videoassisted thoracoscopic repair of an intercostal pulmonary hernia. Surg Endosc 1999;13:166-7.

10. Fiscon V, Portale G, Frigo F, et al. Thoracoscopic repair of lung herniation following minimally invasive cardiothoracic surgery. Chir Ital 2009;61:261-3.

11. Khalil MW, Masala N, Waller DA, et al. Surgical repair of post-traumatic lung hernia using a video-assisted open technique. Interact Cardiovasc Thorac Surg 2008;7:506-7. 
12. Brown WT, Hauser M, Keller FA. Hernia of the lung repaired by VATS: a case report. J Laparoendosc Surg 1996;6:427-30.

Cite this article as: Chiang TY, Yin MF, Yang SM, Chen KC. Thoracoscopic management of incarcerated lung herniation after blunt chest trauma: a case report and literature review. J Thorac Dis 2017;9(3):E253-E257. doi: 10.21037/jtd.2017.03.41
13. Hebra A, Cina R, Streck C. Video-assisted thoracoscopic repair of a lung hernia in a child. J Laparoendosc Adv Surg Tech A 2011;21:763-5. 